\title{
ERRATUM
}

\section{Development of a Reference Material for Image Sharpness Evaluation in Scanning Electron Microscopy - ERRATUM}

Kazuhiro Kumagai and Akira Kurokawa

doi:http://dx.doi.org/10.1017/S1431927616003093, Published by Cambridge University Press, 25 July 2016.

"Development of a Reference Material for Image Sharpness Evaluation in Scanning Electron Microscopy" by Kumagai and Kurokawa originally published with a typographical error in Kazuhiro Kumagai's name. Kazuhiro Kumagai's name has since been corrected online. We apologize for the error.

\section{References}

[1] Kumagai and Kurokawa (2016). Development of a Reference Material for Image Sharpness Evaluation in Scanning Electron Microscopy. Microsc Microanal 22, Suppl 3 (2016), pp. 448-449

(doi:10.1017/S1431927616003093). 